\title{
L'APPLICATION DU THÉORÈME DE BERNOULLI DANS LES ZONES DE DÉCOLLEMENT
}

\section{PAR R. CHEVRAY *}

\section{THE APPLICATION OF THE BERNOULLI THEOREM
IN ZONES OF SEPARATION OF THE BERNOULLI THEOREM
IN ZONES OF SEPARATION}

\section{Introduction}

Les caractéristiques des écoulements avec décollement ont, depuis des années, fait l'objet de recherches analytiques et expérimentales. Bien qu'importantes du point de vue de leurs applications aussi nombreuses qu'essentielles, il est étonnant de constater que les connaissances acquises sur de telles zones de décollement en soient encore à un stade quasi élémentaire.

Une zone de décollemeni peut être décrite en considérant un écoulement primaire et un écoulement secondaire ou induit, chacun étant intimement lié à l'autre par l'intermédiaire d'une zone dans laquelle les tensions tangentielles sont élevées. Le rôle joué par une telle zone de transition est d'un intérêt fondamental, car c'est à l'intérieur même de cette zone qu'a lieu la partie la plus importante de la production de turbulence, caractéristique de tels écoulements.

Ces quinze dernières années ont connu un travail de recherche considérable sur les zones de décollement, travail entrepris sous la direction du professeur H. Rouse à l'Institut de Recherches Hydrauliques d'Iowa.

Débutant avec la thèse de doctorat de H. C. Hsu [1] sur un élargissement brusque de conduite à deux dimensions, plusieurs études se sont succédé. La première d'entre elles, fut une thèse de « Master

* Research Associate, Iowa Institute of Hydraulic Research, Iowa City, Iowa (U.S.A.). 
of Science » présentée par M. Arie [2] sur l'écoulement autour d'une plaque perpendiculaire en contact avec une paroi continue. La seconde et la troisième furent deux thèses de «Master of Science» sur les caractéristiques de la turbulence dans le ressaut hydraulique, présentées par S. Nagaratnam [3] et T. T. Siao [4], rassemblées et discutées dans la référence [5]. La quatrième fut une thèse de doctorat par M.C. Chaturvedi [6] sur des élargissements de conduites avec différentes valeurs de l'angle d'élargissement. Enfin, peu de temps après cette dernière, ce fut une thèse de doctorat de $T$. Carmody [7] sur l'étude d'un disque immergé.

Le but essentiel de toutes ces études a été de mesurer les caractéristiques moyennes aussi bien que turbulentes et d'en établir une corrélation ensuite, au moyen des équations de la quantité de mouvement et de l'énergie. Par les équations de la quantité de mouvement et de l'énergie citées ci-dessus, les caractéristiques du champ de forces et de l'écoulement ont été évaluées. Cependant, par la méthode d'intégration utilisée pour les différentes sections le long de l'écoulement, tous les détails internes disparaissent. Une analyse «ligne par ligne» plutôt que «section par section» semblait alors logique.

A la lumière des différentes publications de Rouse $[8,9,10]$, les termes du théorème de Bernoulli pour un écoulement turbulent ont maintenant été évalués dans la présente étude pour un écoulement normal à une paroi, pour des élargissements de conduite et pour l'écoulement autour d'un disque. Les raisons de ce choix sont doubles. D'abord, loin d'être différents, les écoulements dans des élargissements de conduite sont en beaucoup de points semblables à ceux que l'on rencontre dans l'étude des corps immergés; tandis que les pertes de charge sont considérées pour le premier, sa contrepartie, la résistance est considérée pour le dernier, tous les deux étant la conséquence directe du tourbillon. Ensuite, les mesures détaillées des caractéristiques de l'écoulement moyen ainsi que celles de I'écoulement turbulent étaient disponibles pour une telle analyse dans les études de Arie, Chaturvedi et Carmody.

L'étude analytique présentée ici est une tentative pour obtenir les caractéristiques détaillées du tourbillon dans chacun des cas. Plus que d'être une simple vérification d'une analyse globale, le théorème de Bernoulli pour un écoulement turbulent, considéré comme un moyen de vérifier les mesures obtenues, sera sous certaines hypothèses un moyen appréciable pour la détermination de la tension tangentielle de turbulence en grandeur et position.

\section{Analyse du problème}

\section{ASPECT PHYSIQUE :}

Le décollement dans un écoulement fluide se produit soit le long d'une paroi rectiligne ou progressivement courbée en présence d'un gradient de pression contraire, soit à un changement brusque de géométrie de la paroi. L'écoulement résultant du décollement est par nature non uniforme. $\mathrm{Si}$, de plus, la géométrie des parois restant inchangée, le nombre de Reynolds considéré est tel que l'écoulement devienne turbulent, il sera aussi non per-
[3] and T. T. Siao [4], and discussed and gathered in Reference $[5]$; the fourth was a doctoral study carried out by M. C. Chaturvedi [6] on pipe transitions with different values of the expansion angle, this latter being followed shortly after by the doctoral dissertation by $T$. Carmody [7] on a disk fully surrounded by fluid.

All of these projects have been much concerned with measuring the characteristics of the mean flow and of the turbulence and then correlating them in accordance with the momentum and energy equations. Through the aforementioned momentum and energy equations, the characteristics of the force field and of the flow have been evaluated. However, by the method of integration over sections, all the internal details were necessarily lost. A "line by line" analysis thus seemed necessary.

In the light of Rouse's papers $[8,9,10]$, the pertinent terms of the Bernoulli theorem for turbulent flow have now been evaluated line by line for flow past a normal wall, for flow in pipes with $90^{\circ}$ and $15^{\circ}$ expansions, and for flow around a circular disk. The reasons for the above choices are twofold. First, far from being different, flow in a pipe expansion is very much the same as flow past an immersed body in that, whereas the head loss is considered for the former, its counterpart the drag is considered for the latter, both being the direct consequence of a standing eddy. Second, the detailed data of the mean flow together with those of the turbulence were readily at hand for such an analysis in the work of Arie, Chaturvedi, and Carmody.

The present analytical work is an attempt to obtain the detailed characteristics of the stable eddy in each case. More than a mere check of the overall analysis, the Bernoulli theorem for turbulent flow, as a check on possible local discrepancy, will be under certain assumptions a valuable tool in the determination of both the magnitude and distribution of the turbulent shear.

\section{Physical Aspectrs:}

\section{Analysis of the problem}

Separation in fluid flow is expected to occur either along a straight or smoothly curved boundary in the presence of an adverse preassure gradient and boundary shear, or at an abrupt change in boundary geometry. Flow resulting from separation is nonuniform in nature. If, moreover, the boundary geometry being unchanged, the Reynolds number under consideration is such that turbulence will occur, the flow will also be unsteady, at 
manent, du moins pour autant que l'on considère le mouvement turbulent.

La présente étude illustre un cas typique de décollement apparaissant à un changement brusque de la géométrie de la paroi (à l'arête d'un disque et derrière une paroi perpendiculaire à l'écoulement et au début d'un élargissement brusque de conduite). Sans décollement, la ligne de courant au voisinage de telles arêtes devrait avoir une courbure de valeur infinie, ce qui entraìnerait une valeur infinie du gradient de pression. Cette situation irréelle nous conduit à la conclusion que le décollement doit se produire. En fait, si le rayon de courbure de la ligne de courant de séparation est petit, comme dans le cas du disque ou de la paroi verticale, le point où le décollement se produit sera un point où règnera une pression faible. Tout écoulement avec décollement, au moins dans les cas à deux ou trois dimensions, est étroitement lié à la formation d'une zone de courant de retour contenue par le tourbillon.

A l'intérieur du tourbillon et de la zone de diffusion, l'écoulement est essentiellement rotationnel mais, malheureusement pour l'analyse mathématique, avec une distribution inconnue de la vorticité [9]. En dehors de la zone de mélange dû à la turbulence, la valeur de la somme des énergies cinétique et de pression est approximativement constante, montrant ainsi des caractéristiques d'irrotationalité (l'écoulement étant présumé être irrotationel en amont du point de décollement). La zone de séparation du tourbillon, d'autre part, est caractérisée par un gradient de vitesse élevé dans la direction radiale, qui à son tour provoque une intensité élevée de tension tangentielle turbulente. A l'aval, cette tension joue le double rôle d'accélérer le fluide dans le tourbillon et simultanément de le ralentir dans le courant principal. A l'aval du niveau du tourbillon, par transfert d'énergie, cette tension rend l'écoulement plus uniforme, réduisant la vitesse dans la partie supérieure et l'augmentant dans la partie inférieure.

\section{Formulation ET INTERPRÉTATION DES ÉQUATIONS FONDAMENTALES :}

L'auteur a utilisé, dans les paragraphes suivants, les équations dérivées ainsi que les termes employés dans sa référence [8]. En prenant comme base le système de coordonnées cylindriques défini par $x$, $r$ et $\theta$, dans les directions axiale, radiale et angulaire respectivement, soit $u, v, w$ les composantes respectives de la vitesse.

Pour le cas du disque et de l'élargissement de conduite, les équations de mouvement et de continuité peuvent être simplifiées par le fait que l'écoulement moyen est permanent d'une part et que l'écoulement est axisymétrique d'autre part (la composante tangentielle de la vitesse moyenne, de même que les dérivées partielles par rapport à $\theta$ des quantités moyennes et des quantités dont on a pris la moyenne sont identiquement nulles).

Dans ces conditions, et en séparant les composantes de la vitesse en leurs parties moyennes et de fluctuation : least as far as the turbulent motion is concerned.

The present thesis illustrates a typical case of separation at an abrupt change in boundary geometry (i.e., at the edge of the circular disk, at the edge of the wall, and at the beginning of the abrupt expansion). Without separation the streamline at the edge should have an infinite curvature accompanied by an infinite pressure gradient. This unrealistic situation leads to the conclusion that separation must occur. As a matter of fact, if the radius of curvature of the limiting streamline is small, as in the case of the disk or of the wall, the initial separation point will possess a low pressure.

Flow with separation, in two-dimensional and axisymmetric cases at least, is closely associated with the formation of a zone of reverse flow embodying the standing eddy. Within the eddy and the diffusion zone, the flow is essentially rotational but, unfortunately for a mathematical analysis, with an unknown distribution of vorticity [9]. Outside the zone of turbulent mixing, the flow is seen to retain approximately a constant sum of pressure and velocity head, thus showing irrotafional characteristics (the oncoming flow presumably being irrotational). The border of the eddy, on the other hand, is characterized by a high velocity gradient in the radial direction, which in turn causes a high intensity of shear. Farther downstream, this shear plays over the whole pattern of the flow the twofold role of accelerating the fluid in the eddy and simultaneously decelerating that in the main stream. Past the zone of the eddy, through transfer of energy, the shear will be responsible for rendering the flow more uniform, reducing the velocity in the upper part and increasing it in the lower part.

$$
\left(u=\bar{u}+u^{\prime}, \quad v=\bar{v}+v^{\prime}, \quad w=\bar{w}+w^{\prime}\right)
$$

Formulation AND INTERPRETATION OF THE BASIC EQUATIONS :

The writer has made intensive use in the following paragraphs of the equations derived and of the technical terms used in Reference [8].

With reference to the cylindrical coordinate system defined by $x, r$, and $\theta$ in the axial, radial, angular directions, respectively, let $u, v$, and $w$ be the corresponding components of the velocity. For the cases of the circular disk and of the pipe expansion, the equations of motion and of continuity can be simplified by the fact that the mean flow is steady on the one hand that the flow is axisymmetric on the other hand (i.e., the mean tangentiel velocity component, together will all the partial derivatives with respect to $\theta$ of all averaged quantities, is identically nil).

Under these conditions, and by separating the velocity components into their usual mean and fluctuating parts (i.e., $u=\bar{u}+u^{\prime}, v=\bar{v}+v^{\prime}, w=\bar{w}$ 
les équations de continuité pour le mouvement moyen et turbulent s'écrivent :

$\left.+w^{\prime}\right)$, the continuity equations for mean and turbulent motion can be expressed as:

$$
\begin{gathered}
\frac{\partial(r \bar{u})}{\partial x}+\frac{\partial(r \bar{v})}{\partial r}=0 \\
\frac{\partial\left(r u^{\prime}\right)}{\partial x}+\frac{\partial\left(r v^{\prime}\right)}{\partial r^{\prime}}+\frac{\partial\left(r w^{\prime}\right)}{r \partial \theta}=0
\end{gathered}
$$

tandis que les équations de mouvement s'écrivent $[8$, éq. (14)] : whereas the equations of motion assume the following form [8, Eq. (14)]:

$$
\begin{gathered}
\rho \frac{\partial u}{\partial t}+\rho\left(u \frac{\partial u}{\partial x}+\bar{v} \frac{\partial u}{\partial r}+w \frac{\partial v}{r \partial \theta}\right)=-\frac{\partial}{\partial x}(p+\rho \Omega)+\mu\left(\frac{\partial^{2} u}{\partial x^{2}}+\frac{\partial^{2} u}{\partial r^{2}}+\frac{1}{r} \frac{\partial u}{\partial r}+\frac{\partial^{2} u}{r^{2} \partial \theta^{2}}\right) \\
\rho \frac{\partial v}{\partial t}+\rho\left(u \frac{\partial v}{\partial x}+v \frac{\partial v}{\partial r}+w \frac{\partial v}{r \partial \theta}-\frac{w^{2}}{r}\right) \\
=-\frac{\partial}{\partial r}(\bar{p}+\rho \Omega)+\mu\left(\frac{\partial^{2} v}{\partial x^{2}}+\frac{\partial^{2} v}{\partial r^{2}}+\frac{1}{r} \frac{\partial v}{\partial r}-\frac{v}{r^{2}}+\frac{\partial^{2} v}{r^{2} \partial \theta^{2}}-\frac{2}{r} \frac{\partial w}{r \partial \theta}\right)
\end{gathered}
$$

$\rho\left(u \frac{\partial w}{\partial x}+v \frac{\partial w}{\partial r}+w \frac{\partial w}{r \partial \theta}+\frac{v w}{r}\right)+\rho \frac{\partial w}{\partial t}$

$$
\left.=-\frac{\partial}{r \partial \theta}(p+\rho \Omega)+\mu \frac{\partial^{2} w}{\partial x^{2}}+\frac{\partial^{2} w}{\partial r^{2}}+\frac{1}{r} \frac{\partial w}{\partial r}-\frac{w}{r^{2}}+\frac{\partial^{2} w}{r^{2} \partial \theta^{2}}+\frac{2}{r} \frac{\partial v}{r \partial \theta}\right)
$$

Si la pression décomposée en valeur moyenne et fluctuation $\left(p=\bar{p}+p^{\prime}\right)$, aussi bien que les différentes composantes instantanées de la vitesse sont introduites dans l'équation [3], après avoir pris les moyennes temporelles, nous obtiendrons les équations de Reynolds [8, éq. (16)].
If the pressure, expanded in terms of its mean and fluctuating parts (i.e., $p=\bar{p}+p^{\prime}$ ), as well as the different instantaneous component of the velocity, is introduced into Equation (3), after averaging with respect to time the Reynolds equations will be obtained $[8, \mathrm{Eq} .(16)]$ :

$$
\begin{gathered}
\rho\left(\bar{u} \frac{\partial \bar{u}}{\partial x}+\bar{v} \frac{\partial \bar{u}}{\partial r}+\overline{u^{\prime} \frac{\partial u^{\prime}}{\partial x}}+\overline{v^{\prime} \frac{\partial u^{\prime}}{\partial r}}+\overline{w^{\prime} \frac{\partial u^{\prime}}{r^{\prime} \partial \theta}}\right)=-\frac{\partial}{\partial x}(\bar{p}+\rho \Omega)+\mu\left(\frac{\partial^{2} \bar{u}}{\partial x^{2}}+\frac{\partial^{2} \bar{u}}{\partial r^{2}}+\frac{1}{r} \frac{\partial \bar{u}}{\partial r}\right) \\
\rho\left(\bar{u} \frac{\partial \bar{v}}{\partial x}+\bar{v} \frac{\partial \bar{v}}{\partial r}+\overline{u^{\prime} \frac{\partial v^{\prime}}{\partial x}}+\overline{v^{\prime} \frac{\partial v^{\prime}}{\partial r}}+\overline{w^{\prime} \frac{\partial v^{\prime}}{r \partial \theta}}-\overline{\frac{w^{\prime 2}}{r}}\right) \\
=-\frac{\partial}{\partial r}(\bar{p}+\rho \Omega)+\mu\left(\frac{\partial^{2} \bar{v}}{\partial x^{2}}+\frac{\partial^{2} \bar{u}}{\partial r^{2}}+\frac{1}{r} \frac{\partial \bar{v}}{\partial r}-\frac{v}{r^{2}}\right)
\end{gathered}
$$

Nous pouvons tirer immédiatement de l'équation (4) l'équation d'énergie pour le mouvement moyen; après multiplication de chacune des équations (4) et après addition de ces trois équations, nous obtiendrons : [8, éq. (26)].
Readily obtainable from Equation (4) is the workenergy equation for the mean motion; multiplication of each of Equation (4) by the corresponding mean velocity component and then addition of the three equations will give $[8, \mathrm{Eq} .(26)]$ :

$$
\begin{aligned}
& -\bar{u} \frac{\partial}{\partial x}(\bar{p}+\rho \Omega)-\bar{v} \frac{\partial}{\partial r}(\bar{p}+\rho \Omega) \\
& +\frac{\mu}{r}\left\{\frac{\partial}{\partial x} r\left[2 \bar{u} \frac{\partial \bar{u}}{\partial x}+\bar{v}\left(\frac{\partial \bar{u}}{\partial r}+\frac{\partial \bar{v}}{\partial x}\right)\right]+\frac{\partial}{\partial r}\left[\bar{u}\left(\frac{\partial \bar{u}}{\partial r}+\frac{\partial \bar{v}}{\partial x}\right)+2 \bar{v} \frac{\partial \bar{v}}{\partial r}\right]\right\} \\
& -\frac{\rho}{r}\left[\frac{\partial}{\partial x} r\left(\bar{u} \overline{u^{\prime 2}}+\bar{v} \overline{u^{\prime} v^{\prime}}\right)+\frac{\partial}{\partial r} r\left(\bar{u} \overline{u^{\prime} v^{\prime}}+\bar{v} \overline{\left.v^{\prime 2}\right)}\right]\right. \\
& -\mu\left[2\left(\frac{\partial \bar{u}}{\partial x}\right)^{2}+2\left(\frac{\partial \bar{v}}{\partial r}\right)^{2}+2\left(\frac{\bar{v}}{r}\right)^{2}+\left(\frac{\partial \bar{u}}{\partial r}+\frac{\partial \bar{v}}{\partial x}\right)^{2}\right] \\
& +\rho\left[\overline{u^{\prime 2}} \frac{\partial \bar{u}}{\partial x}+\overline{v^{\prime 2}} \frac{\partial \bar{v}}{\partial r}+\overline{w^{\prime 2}} \frac{\bar{v}}{r}+\overline{u^{\prime} v^{\prime}}\left(\frac{\partial \bar{u}}{\partial r}+\frac{\partial \bar{v}}{\partial x}\right)\right]=\frac{\rho}{2}\left(\bar{u} \frac{\partial \bar{V}^{2}}{\partial x}+\bar{v} \frac{\partial \bar{v} \cdot}{\partial r}\right)
\end{aligned}
$$


La signification de tous les termes contenus dans cette équation sont d'un intérêt primordial, car de cette manière une vue plus approfondie du mécanisme fondamental du mouvement fluide peut être obtenue. Cette équation a été arrangée de facon à montrer dans les termes de gauche la variation du travail fait et dans ceux de droite la variation correspondante de l'énergie cinétique moyenne. Le premier terme dans le membre de gauche représente le travail total fait par les forces de pression et les forces de pesanteur; le second, le travail conservatif fait par les tensions visqueuses; le troisième, le travail total fait par les tensions de Reynolds; le quatrième, le travail fait de manière dissipative par les tensions visqueuses, et le cinquième, le travail fait par les tensions de Reynolds en produisant la turbulence. Par suite, ce terme représente une perte pour le mouvement moyen en mème temps qu'un gain pour le mouvement turbulent. C'est ensuite seulement en examinant sa contrepartie dans l'équation d'énergie pour la turbulence qu'on peut comprendre plus en détail les différentes étapes du mécanisme de la turbulence.

L'équation d'énergie pour les fluctuations turbulentes seules peut être établie en multipliant chacune des équations de Navier et Stokes (éq. (3)) par les composantes respectives de la vitesse, séparées en parties moyennes et de fluctuations. Ensuite, après avoir pris la moyenne temporelle des différents termes, et après avoir soustrait l'équation d'énergie (éq. (5)), nous avons : [8, éq. (27)].
The significance of all the terms embodied in this equation is of primary interest, for in this way a deeper insight into the basic mechanism of finid motion can be gained. This equation has been arranged in such a way as to show on the left-hand side the rate of work done and on the right-hand side the corresponding rate of change of mean linetic energy. The first term in the member on the left represents the total work done by pressure and body forces; the second, the conservative work done by the viscous stresses; the third, the total work done by the Reynolds stresses; the fourth, the work done, in a dissipative way, by the viscous stresses; and the fifth, the work done by the Reynolds stresses in producing turbulence. This term, consequently, represents a loss from the mean motion to the turbulent motion. Only by examining its counterpart in the turbulent-energy equation can further understanding of the different steps of the turbulence mechanism be gained. The energy equation for turbulence fluctuations alone can be derived by multiplying each of the Navier-Stokes equations-Equation (3)-by the corresponding velocity components, expanded in their usual mean and fluctuating parts; then, after averaging has been done on the various terms, subtraction from the foregoing mean energy-work equation-Equation (5)-will give [8, Eq. (27)] :

$$
\begin{aligned}
& -\overline{u^{\prime} \frac{\partial p^{\prime}}{\partial x}}-\overline{v^{\prime} \frac{\partial p^{\prime}}{\partial r}}-\overline{w^{\prime} \frac{\partial p^{\prime}}{r \partial \theta}}-\rho\left[\overline{u^{\prime 2}} \frac{\partial \bar{u}}{\partial x}+\overline{v^{\prime 2}} \frac{\partial \bar{v}}{\partial r}+\overline{w^{\prime 2}} \frac{\bar{v}}{r}+\overline{u^{\prime} v^{\prime}}\left(\frac{\partial \bar{u}}{\partial r}+\frac{\partial \bar{v}}{\partial x}\right)\right] \\
& -\frac{\mu}{r}\left\{\frac{\partial}{\partial x} r\left[\overline{u^{\prime}} \frac{\partial u^{\prime}}{\partial x}+\overline{v^{\prime}\left(\frac{\partial u^{\prime}}{\partial r}+\frac{\partial v^{\prime}}{\partial x}\right)}+\overline{w^{\prime}\left(\frac{\partial w^{\prime}}{\partial x}+\frac{\partial u^{\prime}}{r \partial \theta}\right)}\right]\right. \\
& +\frac{\partial}{\partial r} r\left[\overline{u^{\prime}\left(\frac{\partial u^{\prime}}{\partial r}+\frac{\partial v^{\prime}}{\partial x}\right)}+\overline{v^{\prime} \frac{\partial v^{\prime}}{\partial r}}+\overline{w^{\prime}\left(\frac{\partial v^{\prime}}{r \partial \theta}+\frac{\partial w^{\prime}}{\partial r}-\frac{w w^{\prime}}{r}\right)}\right] \\
& +\frac{\partial}{r \partial \theta} r\left[\overline{u^{\prime}\left(\frac{\partial w^{\prime}}{\partial x}+\overline{\frac{\partial u^{\prime}}{r \partial \theta}}\right)}+\overline{v^{\prime}\left(\frac{\partial v^{\prime}}{r \partial \theta}+\frac{\partial w^{\prime}}{\partial r}-\frac{w^{\prime}}{r}\right)}+\overline{\left.w^{\prime}\left(\frac{\partial w^{\prime}}{r \partial \theta}+\frac{v^{\prime}}{r}\right)\right]}\right\} \\
& =\frac{P}{2}\left(\bar{u} \frac{\partial \overline{V^{\prime 2}}}{\partial x}+\bar{v} \frac{\partial \overline{V^{\prime 2}}}{\partial r}+\overline{u^{\prime}} \frac{\partial \mathrm{V}^{\prime 2}}{\partial x}+\overline{v^{\prime}} \frac{\partial \mathrm{V}^{\prime 2}}{\partial r}+\overline{w w^{\prime} \frac{\partial \mathrm{V}^{\prime 2}}{\partial \theta}}\right) \\
& +\mu\left[\overline{\left(\frac{\partial u^{\prime}}{\partial x}\right)^{2}}+\overline{\left(\frac{\partial v^{\prime}}{\partial x}\right)^{2}}+\overline{\left(\frac{\partial w^{\prime}}{\partial x}\right)^{2}}+\overline{\left(\frac{\partial u^{\prime}}{\partial r}\right)^{2}}+\overline{\left(\frac{\partial v^{\prime}}{\partial r}\right)^{2}}+\left(\overline{\left.\frac{\partial w^{\prime}}{\partial r}\right)^{2}}+\overline{\left(\frac{\partial u^{\prime}}{r \partial \theta}\right)^{2}}+\overline{\left(\frac{\partial v^{\prime}}{r \partial \theta}\right)^{2}}+\left(\overline{\left.\frac{\partial w^{\prime}}{r \partial \theta}\right)^{2}}\right]\right.\right.
\end{aligned}
$$

On peut distinguer successivement dans cette équation les termes représentant: le travail fait par les fluctuations de pression, le transfert d'énergie à partir du mouvement moyen et le travail total fait par les tensions visqueuses dans le membre de gauche. Dans le membre de droite, on reconnaît la variation de l'énergie cinétique de la turbulence dans les termes qui représentent la convection forcée par le mouvement moyen et la diffusion par la turbulence, tandis que le dernier terme est la source principale de perte de l'énergie turbulente par dissipation visqueuse dans les tourbillons. Cependant, puisque les différents termes de l'écquation (6) ne sont pas encore mesurables, cette équation sera essentiellement d'un intérêt théorique dans cette étude.
Successively, in this equation one can distinguish terms for: work done by the pressure fluctuations, transfer of energy from the mean motion, and total work by the viscous stresses on the left-hand side. On the right-hand side, one can recognize the rate of change of the turbulent kinetic energy in terms which represent forced convection by the mean motion and diffusion by the turbulence, whereas the last term is the main source of loss of the turbulent energy by viscous dissipation within the eddies. However, because the different terms of Equation (6) are note as yet measurable, the equation will be only of theoretical interest in this thesis. 


\section{R. CHEVRAY}

L'Équation de Bernoulli EN ÉcoulemeNT TURBULENT :

L'équation de Bernoulli, dans sa forme habituelle, valable seulement dans le cas d'écoulements non visqueux et incompressibles, peut être établie à partir de l'équation d'énergie pour l'écoulement moyen déjà développée pour le cas d'écoulements visqueux et turbulents.

En posant $B^{\prime}=(1 / 2) \rho V^{2}+\bar{p}+\rho \Omega$ dans laquelle $\mathrm{V}$ est la vitesse moyenne totale, $\bar{p}$ la pression moyenne et $\Omega$ la fonction «potentiel dû̀ à la pesanteur », l'équation (5) peut s'écrire sous la forme :

\section{BERNOULLI EQUATION FOR TURBULENT FLOW:}

The Bernoulli equation, which in its usual form is valid only in the case of nonviscous incompressible flow, can, for convenience, be derived for the case of viscous turbulent flow from the previously stated work-energy equation for the mean flow.

By setting $B^{\prime}=\frac{1}{2} \rho \bar{V}^{2}+\overline{\bar{p}}+\rho \Omega$, where $\bar{V}$ is the total mean velocity; $\bar{p}$ is the mean pressure; and $\Omega$ is the gravitational potential, Equation (5) can be written in the following form:

$$
\begin{aligned}
\bar{u} \frac{\partial \mathrm{B}^{\prime}}{\partial x}+\bar{v} \frac{\partial \mathrm{B}^{\prime}}{\partial r}= & -\frac{\rho}{r}\left[\frac{\partial}{\partial x} r\left(\bar{u} \overline{u^{\prime 2}}+\bar{v} \overline{\left.u^{\prime} v^{\prime}\right)}+\frac{\partial}{\partial r}\left(\bar{u} \overline{u^{\prime} v^{\prime}}+\bar{v} \overline{v^{\prime 2}}\right)\right]\right. \\
& +\rho\left[\overline{u^{\prime 2}} \frac{\partial \bar{u}}{\partial x}+\overline{v^{\prime 2}} \frac{\partial \bar{v}}{\partial r}+\overline{w^{\prime 2}} \frac{\bar{v}}{r}+\overline{u^{\prime 2}}\left(\frac{\partial \bar{u}}{\partial r}+\frac{\partial \bar{v}}{\partial x}\right)\right] \\
& +\frac{\mu}{r}\left[\frac{\partial}{\partial x} r\left[2 \bar{u} \frac{\partial \bar{u}}{\partial x}+\bar{v}\left(\frac{\partial \bar{u}}{\partial r}+\frac{\partial \bar{v}}{\partial x}\right)\right]+\frac{\partial}{\partial r}\left[\bar{u}\left(\frac{\partial \bar{u}}{\partial r}+\frac{\partial \bar{v}}{\partial x}\right)+2 \bar{v} \frac{\partial \bar{v}}{\partial r}\right]\right] \\
& -\mu\left[2\left(\frac{\partial \bar{u}}{\partial x}\right)^{2}+2\left(\frac{\partial \bar{v}}{\partial r}\right)^{2}+2\left(\frac{\bar{v}}{r}\right)^{2}+\left(\frac{\partial \bar{u}}{\partial r}+\frac{\partial \bar{v}}{\partial x}\right)^{2}\right]
\end{aligned}
$$

Après multiplication de chacun des membres de cette équation (7) par le déplacement élémentaire le long d'une ligne de courant et en modifiant le premier membre, on peut l'écrire sous la forme :
After multiplying each side of this Equation (7) by the elementary displacement $d s$ along a streamtube and modifying the first term through rules of calculus, by noting that $\vec{u}=d x / d t$ and $\vec{v}=d r / d t$ on can write:

$$
\left(\bar{u} \frac{\partial \mathrm{B}^{\prime}}{\partial x}+\bar{v} \frac{\partial \mathrm{B}^{\prime}}{\partial r}\right) d s=\overline{\mathrm{V}} d \mathrm{~B}^{\prime}
$$

Dans cette équation $d \mathrm{~B}^{\prime}$ est la différentielle totale de la somme $\left[(1 / 2) \rho \bar{V}^{2}+\overline{\bar{p}}+\rho \Omega\right]$. On peut alors évaluer l'intégrale de l'équation (7) entre deux sections repérées par les indices 1 et 2 pour donner :
Herein $d B^{\prime}$ is the total differential of the sum $\left(\frac{1}{2} p \bar{V}^{2}+\bar{p}+\rho \Omega\right)$. Equation (7), now, can be integrated along a streamtube between two sections indicated by the subscripts 1 and 2 to yield:

$$
\begin{aligned}
& \mathrm{B}_{1}^{\prime}=\mathrm{B}_{2}^{\prime}+\int_{\mathrm{s}_{1}}^{\mathrm{s}_{2}} \frac{\rho}{r \overline{\mathrm{V}}}\left[\frac{\partial}{\partial x} \cdot r\left(\bar{u} \overline{u^{\prime 2}}+\bar{v} \overline{u^{\prime} v^{\prime}}\right)+\frac{\partial}{\partial r} r\left(\bar{u} \overline{u^{\prime} v^{\prime}}+\bar{v} \overline{v^{\prime 2}}\right)\right] d s \\
& -\rho \int_{\mathrm{s}_{1}}^{\mathrm{s}_{2}} \frac{1}{\overline{\mathrm{V}}}\left[\overline{u^{\prime 2}} \frac{\partial \bar{u}}{\partial x}+\overline{v^{2}} \frac{\partial \bar{v}}{\partial r}+\overline{w^{\prime 2}} \frac{\bar{v}}{r}+\overline{u^{\prime 2}}\left(\frac{\partial \bar{u}}{\partial r}+\frac{\partial \bar{v}}{\partial \boldsymbol{x}}\right)\right] d s \\
& -\int_{\mathrm{s}_{I}}^{\mathrm{s}_{2}} \frac{\mu}{\bar{V}_{r}}\left\{\frac{\partial}{\partial x} r\left[2 \bar{u} \frac{\partial u}{\partial x}+\bar{v}\left(\frac{\partial \bar{u}}{\partial r}+\frac{\partial \bar{v}}{\partial x}\right)\right]+\frac{\partial}{\partial r}\left[\bar{u}\left(\frac{\partial \bar{u}}{\partial r}+\frac{\partial \vec{v}}{\partial x}\right)+2 \bar{v} \frac{\partial \bar{v}}{\partial r}\right]\right\} d s \\
& +\mu \int_{s_{1}}^{s_{2}} \frac{1}{\bar{V}}\left[2\left(\frac{\partial \bar{u}}{\partial x}\right)^{2}+2\left(\frac{\partial \bar{u}}{\partial r}\right)^{2}+2\left(\frac{\bar{u}}{r}\right)^{2}+\left(\frac{\partial \bar{u}}{\partial r}+\frac{\partial \bar{v}}{\partial x}\right)^{2}\right] d s
\end{aligned}
$$

Les différents termes de cette équation pourraient être discutés de la même façon que l'ont été ceux de l'équation (5). D'une manière analogue, le terme pour la production de la turbulence aura sa contrepartie sous la forme d'un gain pour l'écoulement turbulent aux dépens de l'écoulement moyen.

Tandis que le théorème de Bernoulli dans la forme ci-dessus est rigoureuse en elle-même, il est évidemment trop complexe pour être utilisé pour
The different terms of this equation have the interpretations already discussed for Equation (5). In a similar fashion, the term for production of turbulence will have its counterpart in the form of a gain for the turbulent flow at the cost of the mean flow.

While the Bernoulli equation in the above form is rigorous in itself, it is too complicated to be readily used for computations. However, the equa- 
les calculs. On peut remarquer que l'équation pourra être grandement simplifiée et en fait rendue plus apte au calcul en notant que dans les cas considérés, les forces dues à la pesanteur ne jouent aucun rôle et que les tensions visqueuses peuvent être négligées en comparaison des tensions de Reynolds pour les nombres de Reynolds élevés dont il est question.

L'équation ainsi simplifiée peut s'écrire : tion can be greatly simplified and, in fact, rendered. more amenable to computation by noting that, in the cases under consideration, the gravitational forces play no role, and the viscous stresses can be neglected in comparison with the Reynolds stresses for the high Reynolds numbers involved. The simplified equation thus assumes the form:

$$
\begin{aligned}
\mathrm{B}_{1}^{\prime}=\mathrm{B}_{22}^{\prime} & +\rho \int_{\mathrm{s}_{1}}^{\mathrm{s}_{2}} \frac{1}{r \overline{\mathrm{V}}}\left[\frac{\partial}{\partial x} r\left(\bar{u} \overline{u^{\prime 2}}+\bar{v} \overline{\left.u^{\prime} v^{\prime}\right)}+\frac{\partial}{\partial r} r\left(\bar{u} \overline{u^{\prime} v^{\prime}}+\bar{v} \overline{v^{\prime 2}}\right)\right] d s\right. \\
& -\rho \int_{\mathrm{s}_{1}}^{\mathrm{s}_{2}} \frac{1}{\overline{\mathrm{V}}}\left[\overline{u^{\prime 2}} \frac{\partial \bar{u}}{\partial x}+\overline{v^{\prime 2}} \frac{\partial \bar{v}}{\partial r}+\overline{w^{\prime 2}} \frac{\bar{v}}{r}+\overline{u^{\prime} v^{\prime}}\left(\frac{\partial \bar{u}}{\partial r}+\frac{\partial \bar{v}}{\partial x}\right)\right] d s
\end{aligned}
$$

Et l'équation correspondante pour le cas à deux dimensions pourrait être établie de la même manière pour donner :
The corresponding equation for the two-dimensional case could be derived in the same way to yield:

\section{Discussion des résultats}

\section{VAleurs prévues :}

Comme premier pas vers la discussion des résultats, l'équation de Bernoulli sous forme adimensionnelle doit être rappelée des chapitres précédents :

$$
\mathrm{B}_{1}=\mathrm{B}_{2}+\mathrm{T}+\mathrm{L}
$$

Dans cette expression, il va sans dire, puisque l'énergie est toujours transférée de l'écoulement moyen vers l'écoulement turbulent et n'est jamais récupérée par l'écoulement moyen, que le terme de production de la turbulence $L$ est toujours positif, tandis que $T$ ne conserve pas obligatoirement un même signe puisqu'il représente un transfert d'énergie d'un tube de courant à un autre.

Comme les autres termes de l'équation de Bernoulli déjà considérée, le terme de transfert a les dimensions d'énergie par unité de volume. $\mathrm{Si}$, de plus, la valeur du transfert est multipliée par le volume par unité de temps $\bar{u} d \mathrm{~A}$ s'écoulant à travers une section et que l'on prenne l'intégrale à travers toute la région de l'écoulement, le travail interne sur les surfaces des éléments s'éliminerait et il resterait seul le travail fait par les tensions de Reynolds sur Ies sections extrêmes.

Cependant, ce fait ne nous fournit pas une vérification supplémentaire puisqu'il s'agit simplement de l'équation d'énergie pour l'écoulement moyen dans sa forme intégrale déjà utilisée et faite précédemment $[2,6,7]$.

La somme des trois termes $\mathrm{B}, \mathrm{L}$ et $\mathrm{T}$, de toute évidence, doit posséder une valeur constante et

\section{Discussion of results}

\section{EXPeCted VAlues:}

As a first step towards discussing the results, the equation of Bernoulli in its nondimensional symbolic form must be recalled from the previous chapter:

$$
\mathrm{B}_{1}=\mathrm{B}_{2}+\mathrm{T}+\mathrm{L}
$$

Herein, needless to say, since energy is always transferred from the mean flow to the turbulent flow and is never recovered by the mean flow, the turbulence-production term $\mathrm{L}$ is always positive, whereas $\mathrm{T}$ is not restricted to one sign, since it represents a transfer of energy from one streamtube to another. Like the other terms of the Bernoulli equation already considered, the transfer term is an expression of energy per unit volume. If, moreover, the value of the transfer is multiplied by the volume per unit time $\vec{u} d \mathrm{~A}$ flowing across a section and integrated across the entire flow region, the internal work on the surfaces of the element will cancel and only the work done by the Reynolds stresses on the end sections could remain. However, this fact does not provide a supplementary check, since it is simply the analysis of the energy equation for the mean flow in its integral form previously performed $[2,6,7]$.

The sum of the three terms B, L, and T, quite obviously, must have a constant magnitude of unity everywhere outside the eddy, for the approaching flow possess this value, Within the eddy, on the other hand, every streamline forms a loop and the Iocus of its origin for the computations has been 
égale à l'unité partout en dehors du tourbillon car l'écoulement extérieur voisin possède cette valeur. A l'intérieur du tourbillon, d'autre part, chaque ligne de courant forme une boucle et son origine pour les calculs a été arbitrairement choisie comme étant la ligne de composante axiale de vitesse nulle. Le long de telles lignes de courant fermées, la somme $\mathrm{B}+\mathrm{L}+\mathrm{T}$ sera par conséquent constante, égale à la valeur Bo que l'on a choisie à son origine. La pression à travers l'écoulement est essentiellement égale à celle régnant en dehors du tourbillon et le terme de pertes $L$ possède une valeur beaucoup plus faible que celle trouvée dans l'écoulement principal. De plus, la somme des termes de Bernoulli, comprenant les termes de transfert et de pertes aura une valeur beaucoup plus faible que celle de l'écoulement principal, à cause de la valeur négative du transfert $\mathrm{T}$.

Il découle immédiatement de ces considérations que le terme de pertes $L$ doit nécessairement être égal au terme de transfert $T$ quand une lighe de courant se referme sur elle-même.

\section{RÉsultats des Calculs :}

Mis à part les cas d'un élargissement de conduite de $15^{\circ}$ de la figure 4 , du disque de la figure 6 et de la paroi perpendiculaire à l'écoulement de la figure 8, les résultats sont en défaut dans les premières sections, tandis qu'ils sont en excès dans les sections suivantes, la seule exception étant le cas de l'élargissement brusque de $90^{\circ}$ présenté dans la figure 2. Ces résultats, si l'on fait l'hypothèse que les caractéristiques moyennes de l'écoulement ont été correctement déterminées dans chacun des cas, montrent que les mesures des tensions de la turbulence sont trop faibles dans les premières sections mais trop importantes dans les sections suivantes. On peut être ainsi amené à penser que les mesures de la turbulence plutôt que les mesures des vitesses et pressions moyennes ont été incorrectement déterminées [7].

\section{CoRRECTION DES RÉsULTATS EXPÉRIMENTAUX :}

Si l'on fait l'hypothèse que les caractéristiques moyennes de l'écoulement, de même que celles des tensions normales, ont été correctement déterminées, une première approximation de la valeur de la tension tangentielle $\overline{u^{\prime} v^{\prime}}$ due à la turbulence est obtenue par un calcul par approximations successives en considérant seulement les termes comprenant $\overline{u^{\prime} v^{\prime}}$ multiplié par un gradient de vitesse dans une direction particulière. Une correction de cette approximation est alors possible de manière à tenir compte de toutes les expressions conlenues dans les termes de pertes et de transfert. Ce calcul a été fait dans cette étude pour le cas du disque.

Les valeurs de la tension tangentielle de turbulence mesurée précédemment [7] sont représentées dans la figure 9 , tandis que ceux correspondant au calcul sont représentés sur la figure 10 . Ces valeurs ne sont pas nécessairement ce qu'elles devraient être, car l'hypothèse que les tensions normales aient été correctement mesurées nẻ peut pas être justifiée.

En fait, il est probable sinon certain que les valeurs mesurées des tensions normales de la turbulence sont trop faibles. En conséquence de quoi, chosen arbitrarily as the line of zero forward velocity. Along such closed streamlines, the sum $\mathrm{B}+\mathrm{L}+\mathrm{T}$ will thereby be a constant, equal to the value $B_{0}$ assumed at its origin. The pressure across the flow is essentially the same as that outside the eddy and, due mainly to the low velocity head inside the eddy, the loss term $L$ is seen to have a much smaller value than that of the primary flow. Moreover, due to a negative value of the transfer term $\mathrm{T}$, the value of the Bernoulli sum, including terms for transfer and loss, will be seen to retain a much lower value than that of the primary flow. Readily seen from these considerations is the fact that the loss term $L$ must necessarily equal the transfer term $\mathrm{T}$ when a streamline closes upon itself.

\section{RESULTS OF THE COMPUTATIONS:}

In the case of the $15^{\circ}$ pipe expansion in Figure 4 , the circular disk of Figure 6 , and the normal wall of Figure 8 , the results are deficient in the first sections, whereas they are in excess in the following sections, the only exception being the case of the $90^{\circ}$ pipe expansion presented in Figure 2. These results, if one assumes that the mean characteristics of the flow have been correctly determined in either case, show that the measurements of the turbulence stresses are too low in the sections but too high in the next sections. Consequently, one may be led to think that the turbulence measurements rather that the mean pressures and velocities were incorrectly determined [7].

\section{CORRECTION OF THE EXPERIMENTAL RESULTS:}

If one assumes that the mean characteristics of the flow, together with the normal stresses, have been correctly determined, a first approximation of the turbulent shear $\overline{u^{\prime} v^{\prime}}$ is obtained from a trialand-error computation by considering only the terms involving $\overline{u^{\prime} v^{\prime}}$ times a gradient of velocity in a particular direction. A correction of this approximation is then possible in order to take into account all the expressions contained in the loss and transfer terms. A computation of this sort has been made for the case of the circular disk.

The values of the turbulent shear measured previously [7] are presented in Figure 9, whereas those required by the analysis are presented in Figure 10. These values are not necessarily correct, for the assumption of correct determination of normal stresses is not justified. Indeed, it is probable, although not certain, that the measured values of the turbulence normal stresses are too low. Consequently, the correct value that the turbulent shear should assume will lie somewhere between the curve of measured values an that of the values required by the analysis.

This trial-and-error computation shows that the 
les valeurs correctes que les tensions tangentielles devraient avoir seraient comprises entre les courbes des valeurs mesurées et de celles requises par l'analyse.

Le calcul par approximations successives montre que le terme de transfert dépend principalement des valeurs du gradient dans la direction radiale du produit de $\overline{u^{\prime} v^{\prime}}$ par $r \bar{u}$ et du gradient dans la direction axiale du produit de $\overline{u^{\prime 2}}$ par $r \bar{u}$. Tandis que le terme de pertes dépend plus des valeurs mèmes de $\overline{u^{\prime} v^{\prime}}$ et de $\overline{u^{\prime 2}}$. En d'autres termes, plus la pente de la courbe représentative du produit de rü par $\overline{u^{\prime} v^{\prime}}$ en fonction de $r$ est grande, plus le transfert d'énergie est important.

En fait, on s'apercoit que le signe de ce transfert est déterminé principalement par le signe de la pente de cette courbe. Le terme de pertes, L, d'autre part, sera toujours positif et proportionnel principalement aux valeurs de la tension tangentielle de turbulence et au gradient de la vitesse axiale moyenne dans la direction radiale.

\section{Application de l'équation}

\section{Applicatron numéripue :}

Le théorème de Bernoulli dérivé dans le chapitre précédent n'est évidemment pas pratique pour les calculs. D'abord, l'intégration le long d'une ligne de courant est très difficile à effectuer. Ensuite, de manière à conserver la généralité des expressions, les équations (9) et (10) doivent être écrites dans leurs formes adimensionnelles.

En introduisant la relation $d s / \bar{V}=d x / \overline{\mathrm{U}}$ dans l'équation de Bernoulli et en divisant par la valeur de l'énergie dans l'écoulement d'approche $\mathrm{U}_{0}{ }^{2} / 2$, il en résulte la forme adimensionnelle : transfer term depends mainly on the value of the gradient in the radial direction of the product of $\bar{u} v^{\prime}$ by $r u$ and on the gradient in the $x$ direction of the product of $\bar{u}^{\prime 2}$ by $r \bar{u}$, whereas the loss term depends more on the values of the $\bar{u}^{\prime} \bar{v}^{\prime}$ and $\bar{u}^{\prime 2}$ themselves. In other words, the greater the slope of $r \bar{u}$ times $\tilde{u}^{\prime} \tilde{v}^{\prime}$ versus $r$, the greater the transfer. of energy. The sign of the transfer term in indeed seen to be determined mainly by the sign of the value of this slope. The loss term, on the other hand, will be always positive and directly proportional, mainly, to the values of the turbulence shear stresses and the gradient of the mean axial velocity in the radial direction.

$$
\begin{aligned}
& \mathrm{B}_{1}=\mathrm{B}_{2}+2 \int_{\mathrm{x}_{1}\left(\mathrm{~s}_{1}\right)}^{\mathrm{x}_{2}\left(\mathrm{~s}_{2}\right)} \frac{1}{\left(r / \mathrm{D}_{0}\right)\left(\bar{u} / \mathrm{U}_{0}\right)}\left[\frac{\partial}{\partial x / \mathrm{D}_{0}} \frac{r}{\mathrm{D}_{0}}\left(\frac{\bar{u} \overline{u^{\prime 2}}}{\mathrm{U}_{0} \mathrm{U}_{0}{ }^{2}}+\frac{\bar{v} \overline{u^{\prime} v^{\prime}}}{\mathrm{U}_{0} \mathrm{U}_{0}{ }^{2}}\right)+\frac{\partial}{\partial r / \mathrm{D}_{0}} \frac{r}{\mathrm{D}_{0}}\left(\frac{\bar{u} \overline{u^{\prime} v^{\prime}}}{\mathrm{U}_{0} \mathrm{U}_{0}{ }^{2}}+\frac{\bar{v} \overline{v^{\prime 2}}}{\mathrm{U}_{0} \mathrm{U}_{0}{ }^{2}}\right] \frac{d x}{\mathrm{D}_{0}}\right. \\
& -2 \int_{\mathrm{x}_{1}\left(\mathrm{~s}_{1}\right)}^{\mathrm{x}_{2}\left(\mathrm{~s}_{2}\right)} \frac{1}{\bar{u} / \mathrm{U}_{0}}\left[\frac{\overline{u^{\prime 2}}}{\mathrm{U}_{0}^{2}} \frac{\partial \bar{u} / \mathrm{U}_{0}}{\partial x / \mathrm{D}_{0}}+\frac{\overline{v^{\prime 2}}}{\mathrm{U}_{0}^{2}} \frac{\partial \bar{v} / \mathrm{U}_{0}}{\partial r / \mathrm{D}_{0}}+\frac{\overline{w^{\prime 2}}}{\mathrm{U}_{0}^{2}} \frac{v / \mathrm{U}_{0}}{r / \mathrm{D}_{0}}+\frac{\overline{u^{\prime} v^{\prime}}}{\mathrm{U}_{0}^{2}}\left(\frac{\partial \bar{u} / \mathrm{U}_{0}}{\partial r / \mathrm{D}_{0}}+\frac{\partial \bar{v} / \mathrm{U}_{0}}{\partial x / \mathrm{D}_{0}}\right)^{-}\right] \frac{d x}{\mathrm{D}_{0}}
\end{aligned}
$$

dans laquelle $B$ est la contrepartie sans dimension de $B^{\prime}, \mathrm{U}_{0}$ la vitesse dans l'écoulement du courant d'approche et $D_{0}$ une longueur caractéristique; dans ce cas, le diamètre de la section d'entrée au début de l'élargissement.

D'une manière très semblable, l'équation de Bernoulli pour un écoulement turbulent à trois dimensions peut ètre établie. L'équation adimensionnelle simplifiée se réduit à :

\section{Application of the equation}

NUMERICAL PROCEDURE:

The Bernoulli equation derived in the preceding chapter is evidently not convenient for computational use for two reasons: first, integration along a stream line is difficult to handle; second, in order to increase the utility of the expressions, Equations (9) and (10) must be written in non-dimensional form.

Upon introducing the relationship $d s / \overline{\mathrm{V}}=d x / \bar{u}$ in the Bernoulli equation and dividing by the value of the energy level in the oncoming flow, $\mathrm{\rho U}_{0}{ }^{2} / 2$, the following dimensionless expression will result:

$$
\begin{aligned}
& \mathrm{B}_{1}=\mathrm{B}_{2}+2 \int_{\mathrm{x}_{1}}^{\mathrm{x}_{2}} \frac{1}{\bar{u} / \mathrm{U}_{0}}\left[\frac{\partial}{\partial x / h}\left(\frac{\bar{u} \overline{u^{\prime 2}}}{\mathrm{U}_{0} \mathrm{U}_{0}^{2}}+\frac{\bar{v} \overline{u^{\prime} v^{\prime}}}{\mathrm{U}_{0} \mathrm{U}_{0}^{2}}\right)+\frac{\partial}{\partial y / h}\left(\frac{\bar{u} \overline{u^{\prime} v^{\prime}}}{\mathrm{U}_{0} \mathrm{U}_{0}^{2}}+\frac{\bar{v} \overline{v^{\prime 2}}}{\mathrm{U}_{0} \mathrm{U}_{0}^{2}}\right] \frac{d s}{h}\right. \\
& -2 \int_{\mathrm{x}_{1}}^{\mathrm{x}_{2}} \frac{1}{\bar{u} / \mathrm{U}_{0}}\left[\overline{\frac{u^{\prime 2}}{\mathrm{U}_{0}{ }^{2}}} \frac{\partial \bar{u} / \mathrm{U}_{0}}{\partial x / h}+\overline{\frac{v^{\prime 2}}{\mathrm{U}_{0}^{2}}} \frac{\partial \bar{v} / \mathrm{U}_{0}}{\partial y / h}+\overline{\frac{u^{\prime} v^{\prime}}{\mathrm{U}_{0}^{2}}}\left(\frac{\partial \bar{v} / \mathrm{U}_{0}}{\partial x / h}+\frac{\partial \bar{u} / \mathrm{U}_{0}}{\partial y / h}\right)\right] \frac{d s}{h}
\end{aligned}
$$

dans laquelle $h$ est une longueur caractéristique choisie comme étant la hauteur d'une paroi.
Herein, $B$ is the dimensionless counterpart of $B^{\prime}$, $\mathrm{U}_{0}$ the velocity in the approaching flow and $\mathrm{D}_{0}$ a characteristic length, in this case the diameter of the intake end of the pipe expansion.

In a very similar way, the Bernoulli equation for two-dimensional turbulent flow can be derived. The simplified dimensionless equation reduces to:

\footnotetext{
choisie compe etant la hauteur d'une paroi.
}

in which $h$ is a characteristic length chosen as the height of the wall. 
Il est à noter que les termes dans les équations (12) et (13) sont des intégrales curvilignes. En d'autres termes, elles sont fonction de l'abscisse curviligne $s / \mathrm{D}_{0}$ ou $s / h$ le long d'une ligne de courant. En conséquence, toutes les quantités ci-dessus ont été calculées dans la présente étude pour tonte la région de l'écoulement en un nombre de points aussi grand que possible. Les valeurs prises par ces fonctions le long d'une ligne de courant ont été tracées en fonction des valeurs correspondantes de $x / \mathrm{D}_{0}$ ou $x / h$ (la longueur de l'are le long d'une ligne de courant étant prise comme fonction des coordonnées $x / \mathrm{D}_{0}$ ou $x / h$ pour l'intégration).

Pour tous les calculs, les deux hypothèses ayant trait aux caractéristiques de la turbulence et qui furent considérées comme justifiées dans les recherches précédentes $(6,7)$ ont été utilisées. Ces hypothèses sont $: \overline{v^{\prime 2}}=\overline{w w^{2}}$ pour des angles de $90^{\circ}$ et $15^{\circ}$ d'élargissement de conduite :

$$
\overline{u^{\prime 2}}=2 \overline{v^{\prime 2}}=\overline{2 w^{\prime 2}}
$$

pour le disque.

\section{Présentation des résultats :}

Les résultats de l'analyse sont présentés sous forme graphique. Les figures $1,3,5$ et 7 montrent les lignes de courant tracées à l'extérieur et à l'intérieur du tourbillon et repérées par les valeurs de la fonction du courant $\psi$. Les différents termes de l'équation de Bernoulli sont représentés dans les figures correspondantes $2,4,6$ et 8 . Ils sont tracés nécessairement d'une manière cumulative puisque ce qui a été perdu ou transféré entre deux sections est évidemment perdu ou transféré pour les sections suivantes.

Dans l'écoulement principal, la somme adimensionnelle de Bernoulli possède une valeur égale à l'unité pour la section d'entrée, cette section a donc été choisie comme origine pour les calculs. Cependant, à l'intérieur du tourbillon, aucune section semblable n'existe; par conséquent, l'intersection de chaque ligne de courant avec la ligne lieu des points de composante axiale de vitesse nulle a été choisie arbitrairement comme origine pour les calculs.
It must be noted that the terms under the integral sign in Equations (12) and (13) are line integrals. In other words, they are functions of the arc length $s / \mathrm{D}_{0}$ or $s / h$ along a streamline. Consequently, all the above quantities have been computed in the present thesis throughout the flow region at as many points as possible. The values taken by these functions along a streamline have then been plotted against the corresponding values of $x / \mathrm{D}_{0}$ or $x / h$ (the arc length along a streamline being a function of the coordinates $x / \mathrm{D}_{0}$ or $x / h$ ) for integration.

Throughout the computations, the two assumptions with regard to the turbulence characteristics which were considered satisfactory by the previous investigator $[6,7]$ were used. They are: $\bar{v}^{\prime 2}=\bar{w}^{\prime 2}$ for $90^{\circ}$ and $15^{\circ}$ pipe expansion; $\bar{u}^{\prime 2}=2 \bar{v}^{\prime 2}=2 w^{\prime 2}$ for the circular disk.

\section{Presentatron of Results:}

The results of the aforementioned analysis are presented in graphical form. Figures $1,3,5$, and 7 show the patters of streamlines extended into the eddy zone, and referred to by the values of the usual stream function $\psi$. The terms of the Bernoulli equation are presented in the corresponding Figures $2,4,6$ and 8 . They are necessarily plotted in a cumulative way, since what has been lost or transferred between two sections is evidently lost or transferred for the following sections.

In the primary flow, the non-dimensional Bernoulli sum assumes a value of unity at the intake section; this section has consequently been chosen as the initial section for the computations. However, within the eddy, no such initial section exists; hence the intersection of every streamline with the line of zero forward velocity has been chosen arbitrarily as origin for the computations.

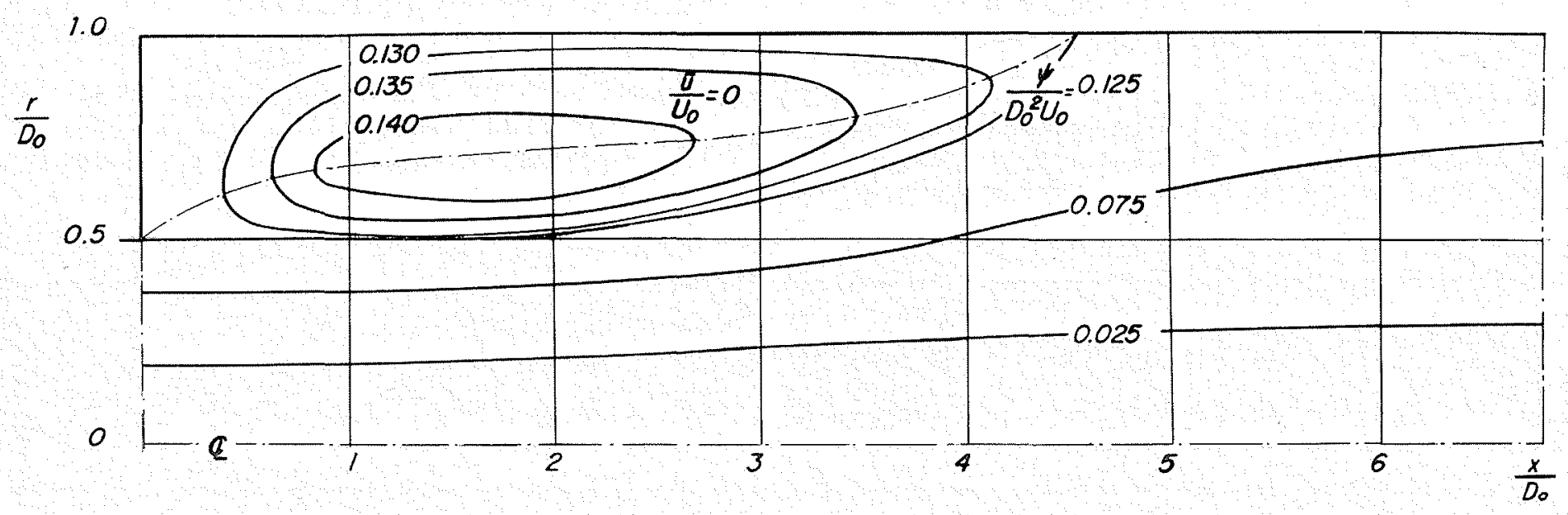

Répartition des filets liquides, pour un tuyau divergent de $90^{\circ} / \mathbf{1} /$ Pattern of stream lines for $90^{\circ}$ pipe expansion 
Dans cette étude, outre une discussion générale de la transformation de l'énergie dans un écoulement avec décollement, la variation des différents termes de l'équation de Bernoulli le long d'une ligne de courant a été présentée. Dans l'analyse mathématique du problème, l'équation de Bernoulli a été dérivée à partir des équations fondamentales du mouvement et ensuite simplifiée pour les quatre cas considérés dans cette étude. Cependant il est à noter qu'aucun renseignement concernant l'énergie cinétique de la turbulence de même que la vraie valeur de l'énergie perdue localement par dissipation visqueuse ne peut être obtenu par cette équation de Bernoulli. Les résultats, montrant les valeurs des différents termes de l'équation de Bernoulli, ont été représentés sous forme de graphique pour différentes sections le long d'une ligne de courant. Il apparaît de par ces graphiques que :

La plus grande partie de l'énergie perdue a lieu dans la région du tourbillon et est due à la production de turbulence.

En considérant uniquement l'écoulement principal, il apparait que le terme représentant le transfert d'énergie est supérieur à celui représentant la production de turbulence montrant ainsi que la plus grande partie de l'énergie est transférée au tourbillon et faisant ressortir par là même le rôle joué par le tourbillon dans les phénomènes de séparation.

La valeur prise par la somme des termes de Bernoulli décroît partout à l'exception de la zone du tourbillon au voisinage de l'écoulement principal où un gain local d'énergie par transfert excède la valeur prise pour la production de turbulence.

L'application du théorème de Bernoulli à un écoulement turbulent est certainement une vérification efficace d'une analyse globaie; elle est un bon moyen par lequel le ròle joué par le tourbillon peut être éclairci et enfin, elle nous fournit sous certaines hypothèses une méthode de détermination de ce que les tensions tangentielles de turbulence devraient être.
In this thesis, a general discussion of the conversion of energy in flow with separation has been presented. More specifically, the variation of the different terms of the Bernoulli equation along a streamline has been studied. In the mathematical analysis of the problem, the Bernoulli equation was derived from the basic equations of motion, and then simplified for the four cases under consideration in this study. However, no information regarding the kinetic energy of the turbulent flow can be gained by this use of the Bernoulli equation, nor is a treu measure obtained of the energy lost locally through viscous dissipation. The results, showing the values of the pertinent Bernoulli terms, have been represented in graphical form for different sections along a streamline. These results lead to the following conclusions:

1. Most of the loss of energy takes place in the region of the eddy and is due in both primary and secondary flow to the production of turbulence.

2. The usual Bernoulli sum, in general, decreases everywhere except in that part of the eddy close to the primary flow, where the local gain of energy through transfer is in excess of the local turbulence production.

3. This application of the Bernoulli theorem for turbulent flow in a region of separation provides, under certain assumptions, a method of determining what the turbulence shear stresses should be; it is certainly an efficient check for an over-all analysis, and it is a good means by which the role of the eddy can be elucidated.

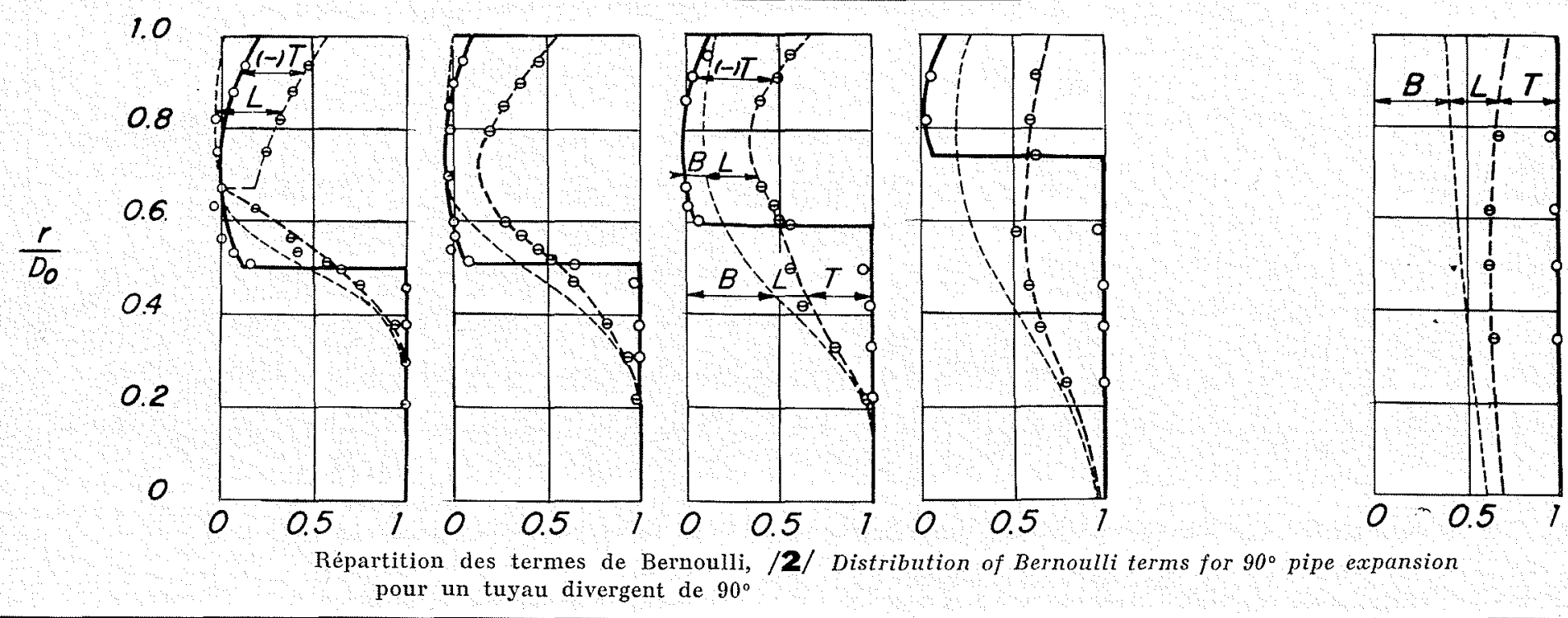




\section{R. CHEVRAY}

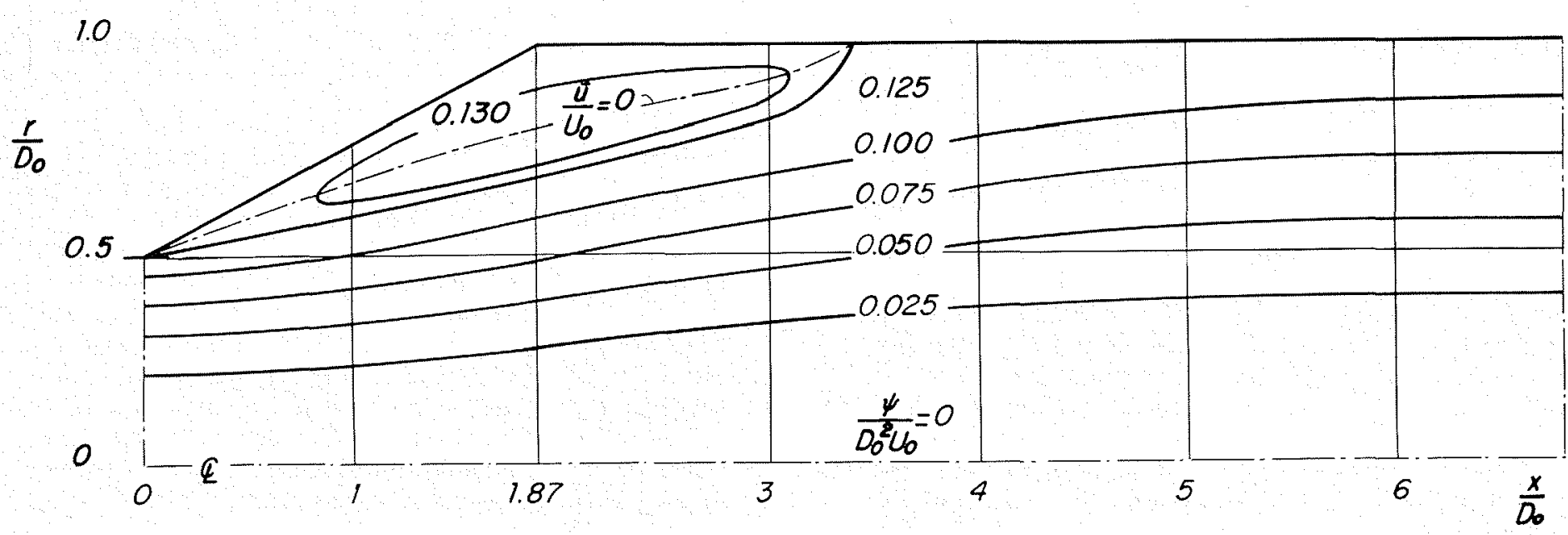

Répartition des filets liquides, pour un tuyau divergent de $15^{\circ} / \mathbf{3} /$ Pattern of stream lines for $15^{\circ}$ pipe expansion

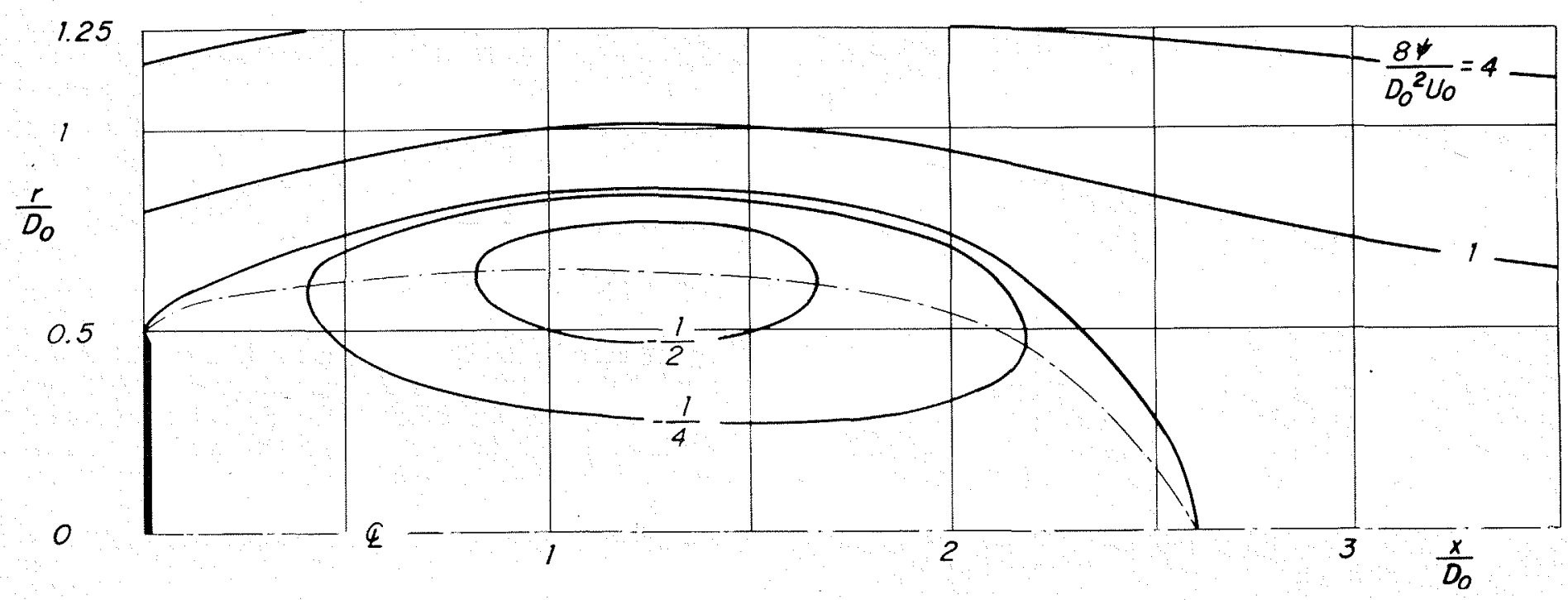

Répartition des filets liquides, pour un disque circulaire /5/ Pattern of stream lines for circular dis:

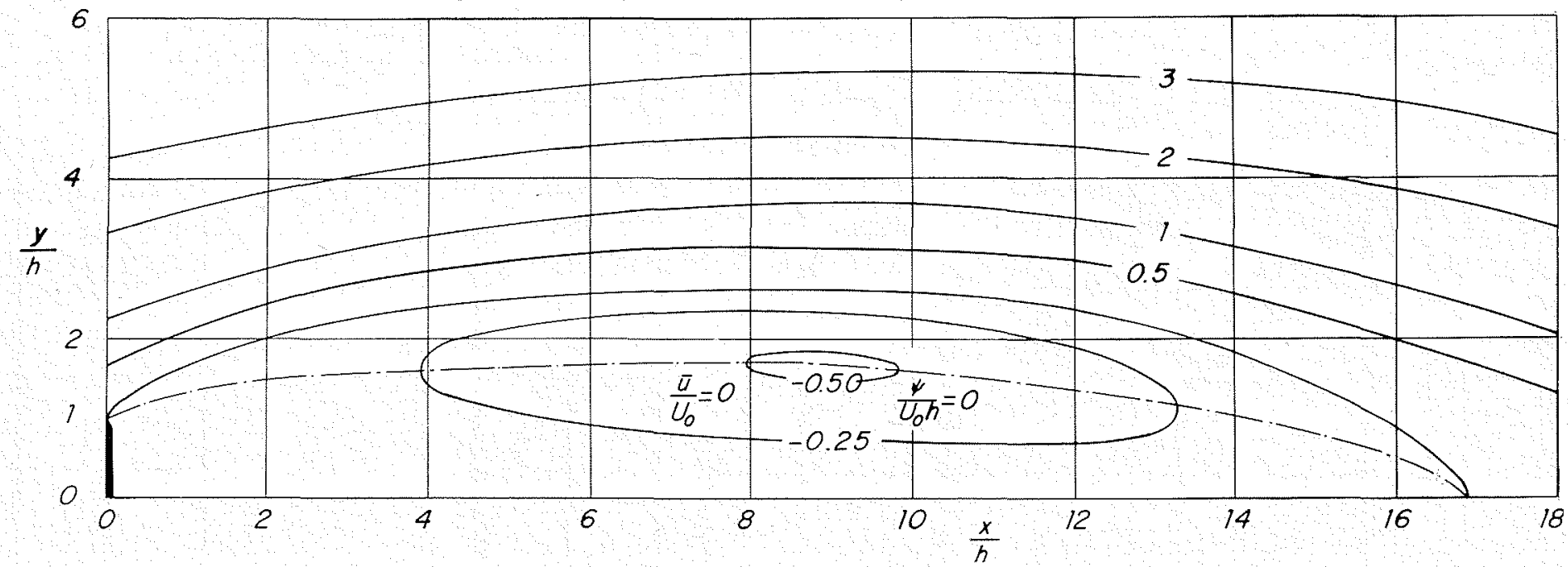

Répartition des filets liquides, pour une paroi normale /7/ Pattern of stream lines for normal wall 

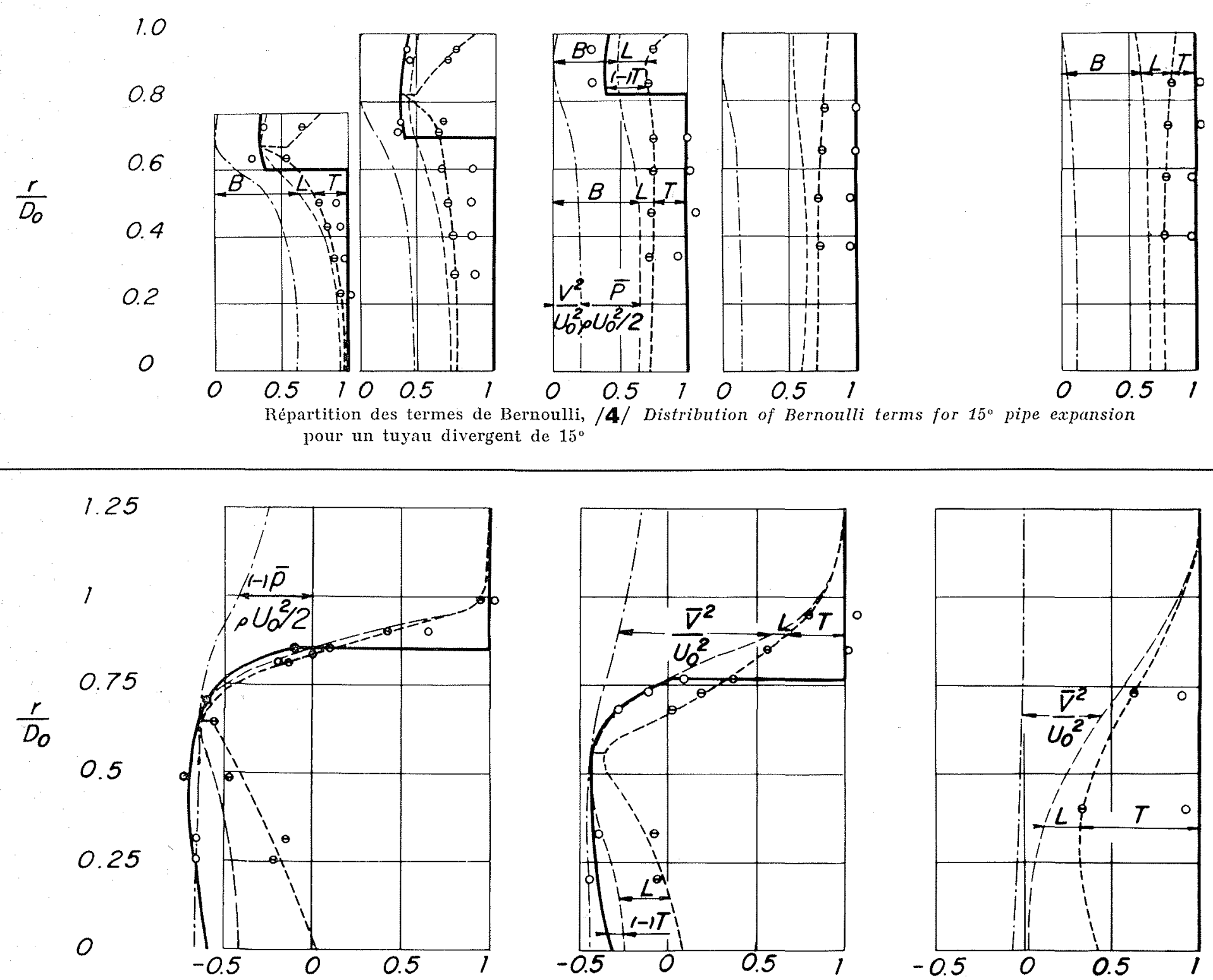

Répartition des termes de Bernoulli, pour un disque circulaire /6/ Distribution of Bernoulli terms for circular disk
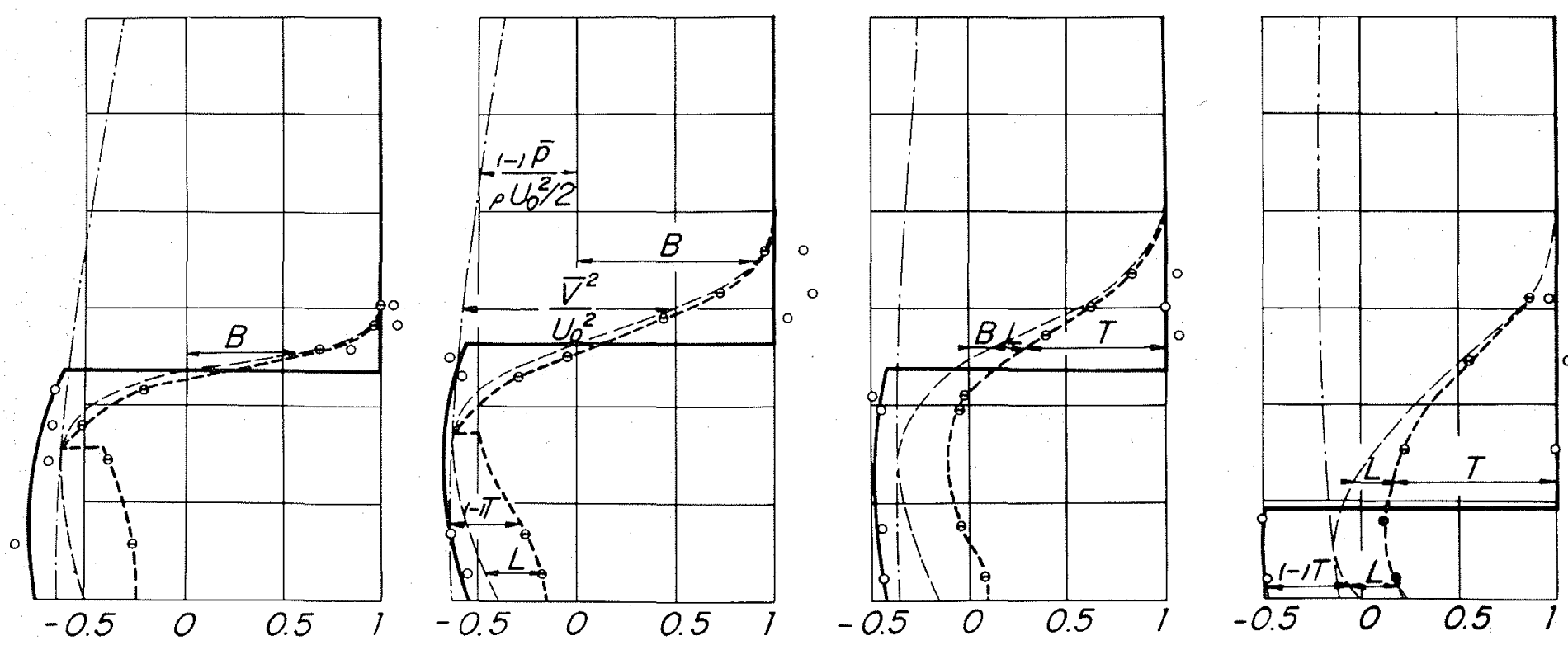

Répartition des termes de Bernoulli, pour une paroi normale /8/ Distribution of Bernoulli terms for normal wall 


\section{R. CHEVRAY}

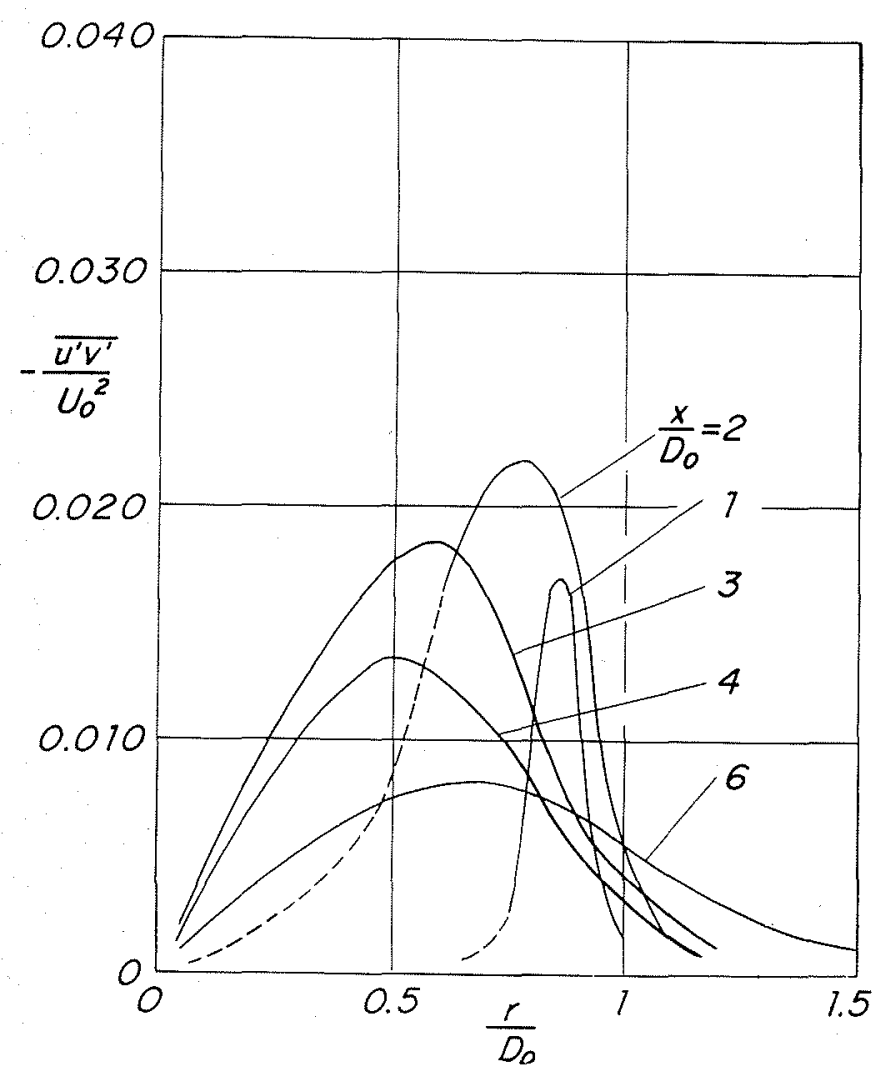

Répartition des valeurs mesurées de l'effort de cisaillement turbulent, pour un disque circulaire, dans les sections correspondant $\dot{a} 1 \leqslant x / D_{0} \leqslant 6$ Distribution of measured turbulence shear stress for circular dis. in sections $1 \leqslant \mathrm{x} / D_{0} \leqslant 6$

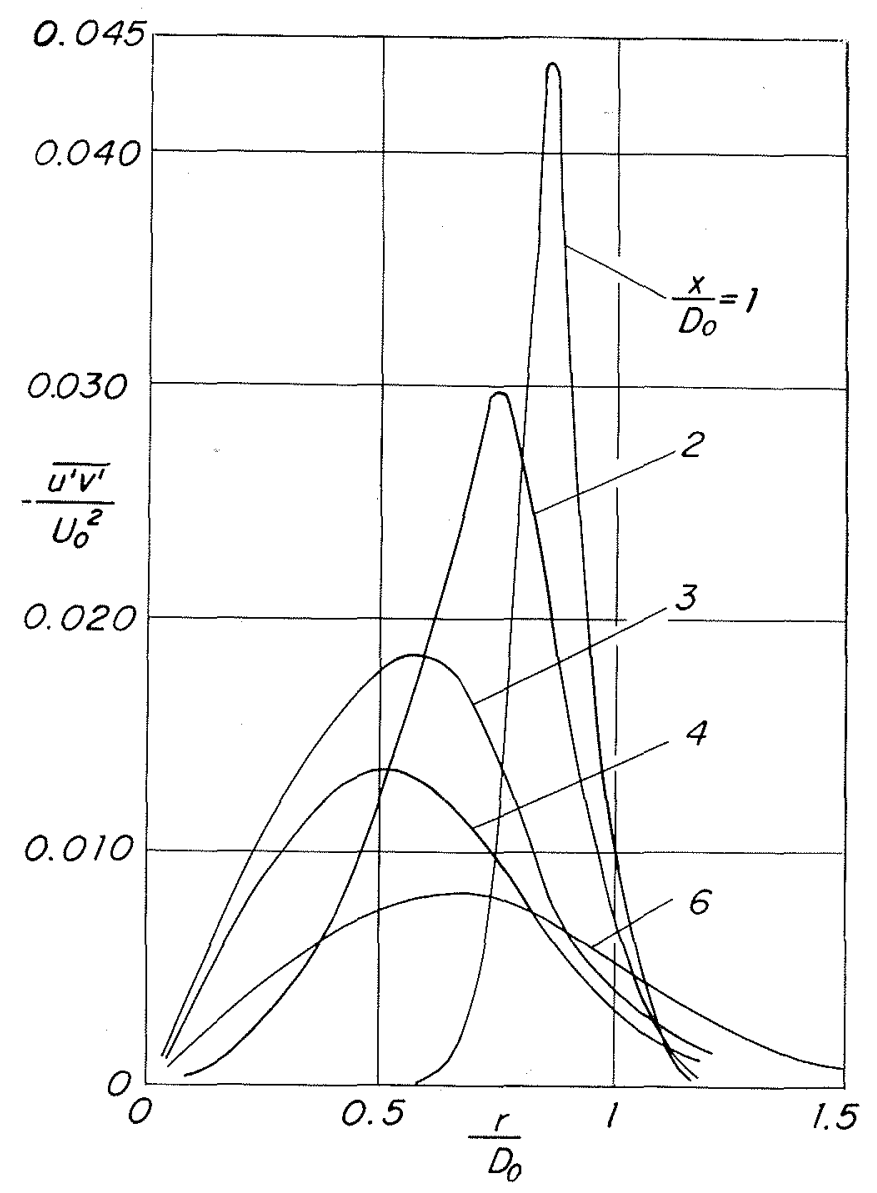

10/ Répartition des valeurs recherchées de l'effort de cisaillement turbulent, pour un disque circulaire, dans les sections correspondant à $1 \leqslant x / \mathrm{D}_{0} \leqslant 6$ Distribution of required turbulence shear stress for circular disk in sections $1 \leqslant \mathrm{x} / D_{0} \leqslant 6$
[1] Hsu (H.C.). - Characteristics of Mean Flow and Turbulence at an Abrupt Two-Dimensional Expansion. Ph. D. Dissertation, State University of Iowa (1950).

[2] Arie (M.). - Flow Past a Normal Plate in Contact with a Boundary. M.S. Thesis, State University of Iowa (1950).

[3] Nagarativam (S.). - The Mechanism of Energy Dissipation in the Hydraulic Jump. M.S. Thesis, State University of Iowa (1957).

[4] SIAO (T.T.). - Characteristics of Turbulence in an Air-Flow Model of the Hydraulic Jump. Ph. D. Dissertation, State University of Iowa (1954).

[5] Rouse (H.), Srao (T.T.) and Nagaratram (S.). - Turbulence Characteristics of the Hydraulic Jump. Proceedings American Society of Civil Engineers (February 1958).
[6] Chaturvedi (M.C.). - Filow Characteristics at Abrupt Axisymmetric Expansions. Ph. D. Dissertation, State University of lowa (1962).

[7] Canmody (J.T.). - Establishment of the Wake behind a Disk. Ph. D. Dissertation, State University of Iowa (1963).

[8] Rouse (H.). - Répartition de l'énergie dans les zones de décollement. La Houille Blanche, no 3 (mai 1960) et $\mathrm{n}^{\circ} 4$ (juin 1960).

[9] Rouse (H.). - Energy Transformation in Zones of Separation. International Association for Hydraulic Research Ninth Convention, Dubrovnil, Yugoslavia (1961).

[10] Rouse (H.) - On the Bernoulli Theorem for Turbulent Flow. Miszellaneen der Angewandten Mechanit, Akademie-Verlag, Berlin (1962), p. 267. 